\title{
A FORM OF EQUATION OF MOTION FOR CHAPLYGHIN'S SYSTEMS
}

\author{
DO SANH \\ Hanoi University of Technology
}

\section{Introduction}

The Chaplyghin's systems play an important role in engineering. There exist some methods for establishing these systems. It is necessary to pay attention to the Chaplyghin's equations which are written by means of the Lagrangian function in independent variables. By this, the mentioned equations are a closed set of second order differential equations. The application of the Lagrangian function for writing equations of motion is an advantageous point of the Chaplyghin's method. However, the algorithm of establishing these equations is complicated enough, especially for the large systems.

In the present paper, a form of equations of motion for the Chaplyghin's systems is introduced. The scheme for writing these equations is very simple, because they are established by means of only the matrix of inertia of Lagrangian function and are written in the matrix form.

It is necessary to emphasize that the equations obtained are very convenient for automatically programming on computers.

\section{The form of equations of motion for Chaplyghin's systems}

Let us consider a mechanical system with $n$ holonomic coordinates $q_{i}$ ( $i=$ $1,2, \ldots, n)$. Assume that the matrix of inertia of the system is denoted by $\mathbf{A}$ which is an $n \times n$ positive define symmetric matrix. The kinetic energy of the system is of the form:

$$
T=\frac{1}{2} \dot{\mathbf{q}}^{T} \mathbf{A} \dot{\mathbf{q}}
$$

where $\dot{\mathbf{q}}$ is an $n \times 1$ matrix of generalized velocities. The letter located on the high right corner denotes the transposition.

The generalized force corresponding to the coordinate $q_{i}$ is denoted by $Q_{i}$ 
$(i=1,2, \ldots, n)$. The $n \times 1$ matrix of generalized forces is denoted by $\mathrm{Q}$, that is:

$$
\mathrm{Q}^{T}=\left\|Q_{1} Q_{2} \ldots Q_{n}\right\| .
$$

Suppose that the system under consideration is subjected to constraints of the form:

$$
\dot{q}_{r}=\sum_{j=1}^{k} b_{r j} \dot{q}_{j}, \quad r=\overline{1, s} ; k=n-s .
$$

Let us consider the case when coefficients $b_{r j}$ do not contain the coordinates $q_{r}$ $(r=1,2, \ldots, s)$. Besides, these coordinates are absent in the elements of the matrix of inertia and in the expressions of generalized forces. Such a system is said to be the Chaplyghin's system. Let us introduce the following notations:

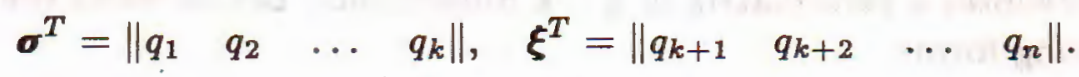

In other words, $\sigma$ is the $k \times 1$ matrix of independent coordinates, but $\xi$ the $s \times 1$ matrix of dependent ones. The constraint equations (2.3) are written in the matrix form as follows:

$$
\dot{\xi}=\mathbf{B} \dot{\boldsymbol{\sigma}},
$$

where the $s \times k$ matrix $\mathrm{B}$ is of the form:

$$
\mathbf{B}=\left\|b_{r j}\right\| .
$$

It is necessary to notice that:

$$
\mathbf{B}=\mathbf{B}(\boldsymbol{\sigma}), \quad \mathbf{A}=\mathbf{A}(\boldsymbol{\sigma}), \quad \mathbf{Q}=\mathbf{Q}[\boldsymbol{\sigma}, \dot{\mathbf{q}}(\boldsymbol{\sigma}, \dot{\boldsymbol{\sigma}})] .
$$

In order to write the equation of motion for the Chaplyghin's system, we apply the equations given in [4]:

$$
\mathbf{D} \mathbf{A} \ddot{\mathbf{q}}=\mathbf{D} \mathbf{Q}+\mathbf{D} \mathbf{G} \text {, }
$$

where $\mathbf{D}$ is the $k \times n$ matrix, the elements of which are the coefficients in the expressions of dependent accelerations in terms of independent ones [2], G is the $n \times 1$ matrix which is determineded by the matrix of inertia $[3,4]$.

Because of (2.7) related to the matrix $\mathbf{A}$, it is easy to show that the matrix $\mathbf{G}$ do not contain dependent coordinates, i.e.

$$
\mathbf{G}=\mathbf{G}[\boldsymbol{\sigma}, \dot{\mathbf{q}}(\dot{\boldsymbol{\sigma}}, \boldsymbol{\sigma})] .
$$


In the case of the Chaplyghin's system the matrix $\mathbf{D}$ could be taken in the form:

$$
\mathbf{D}^{T}=\left\|\mathbf{E} \quad \mathbf{B}^{T}\right\|,
$$

where $\mathbf{E}$ is the identity matrix of $k$ orders, $\mathbf{B}$ is the matrix (2.6).

Because of (2.10), we have [2]

$$
\dot{\mathbf{q}}=\|\dot{\boldsymbol{\sigma}} \quad \dot{\boldsymbol{\xi}}\|=\left\|\begin{array}{l}
\mathbf{E} \\
\mathbf{B}
\end{array}\right\| \dot{\boldsymbol{\sigma}} .
$$

From here we obtain:

$$
\ddot{\mathbf{q}}=\left\|\begin{array}{l}
\mathbf{E} \\
\mathbf{B}
\end{array}\right\| \dot{\boldsymbol{\sigma}}+\left\|\begin{array}{l}
\mathbf{O} \\
\dot{\mathbf{B}}
\end{array}\right\| \dot{\boldsymbol{\sigma}}
$$

in which $\mathbf{O}$ denotes a zero matrix of $k \times k$ dimensions. Let us write the matrix $\mathbf{A}$ in the following form:

$$
\mathbf{A}=\left\|\begin{array}{ll}
\mathbf{A}_{1} & \mathbf{A}_{4} \\
\mathbf{A}_{3} & \mathbf{A}_{2}
\end{array}\right\|,
$$

where

$\mathbf{A}_{1}$ is the matrix of $k$ first rows and columns,

$\mathbf{A}_{2}$ is the matrix of $s$ last rows and columns,

$\mathbf{A}_{3}$ is the matrix of $s$ last rows and $k$ first columns,

$\mathbf{A}_{4}$ is the matrix of $s$ first rows and $k$ last columns.

Similarly, we put

$$
\mathbf{Q}=\left\|\begin{array}{c}
\mathbf{Q}_{\sigma} \\
\mathbf{Q}_{\boldsymbol{\xi}}
\end{array}\right\|, \quad \mathbf{G}=\left\|\begin{array}{l}
\mathbf{G}_{\sigma} \\
\mathbf{G}_{\boldsymbol{\xi}}
\end{array}\right\|,
$$

where $\mathbf{Q}_{\sigma}, \mathbf{G}_{\sigma}$ are of $k$ first rows, but $\mathbf{Q}_{\xi}, \mathbf{G}_{\xi}$ of $s$ last rows of the matrix $\mathbf{Q}$ and $G$ respectively.

In consideration of $(2.11),(2.12),(2.13)$ and $(2.14)$ the equations $(2.8)$ are written in the following form:

$$
\mathbf{A}_{\sigma} \dot{\boldsymbol{\sigma}}=\mathbf{Q}_{\sigma}+\mathbf{G}_{\sigma}+\mathbf{B}^{T}\left(\mathbf{Q}_{\xi}+\mathbf{G}_{\xi}\right)-\mathbf{A}^{*} \dot{\boldsymbol{\sigma}}
$$

where $\mathbf{A}_{\sigma}, \mathbf{A}^{*}$ are the $k \times k$ matrices of the form:

$$
\begin{aligned}
& \mathbf{A}_{\sigma}=\mathbf{A}_{1}+\mathbf{B}^{T} \mathbf{A}_{3}+\mathbf{A}_{4} \mathbf{B}+\mathbf{B}^{T} \mathbf{A}_{2} \mathbf{B} \\
& \mathbf{A}^{*}=\left(\mathbf{A}_{4}+\mathbf{B}^{T} \mathbf{A}_{2}\right) \dot{\mathbf{B}} .
\end{aligned}
$$


By such a way we obtain a closed set of $k$ ( $k$ to be the number of the degrees of freedom of the under consideration) differential equations of second order (2.15) which describe the motion of the Chaplyghin's system.

Let us consider now the case when the constraint equations are written in the unsolved form with respect to dependent velocities. In connection with this, let us investigate the system subjected to the nonholonomic constrains of the form:

$$
\sum_{j=1}^{n} C_{r j} \dot{q}_{j}=0, \quad r=\overline{1, s}
$$

in which the dependent coordinates $q_{r}(r=\overline{1, s}=n-k)$ are absent in the coeffcients

$$
\frac{\partial C_{r j}}{\partial \xi_{\mu}} \equiv 0, \quad j=\overline{1, n} ; \mu, r=\overline{1, s}=n-k .
$$

The constraint equations (2.18) could be written in the form:

$$
\mathrm{C} \dot{\mathbf{q}}=\mathbf{0},
$$

where $\mathbf{C}(\boldsymbol{\sigma})$ is an $s \times n$ matrix.

It is easy to show that it is possible to apply the equations (2.15) for the system under consideration. For this aim, it is necessary to define the matrix $\mathbf{B}$ satisfying the following equations [2]

$$
\left\|\mathbf{E} \quad \mathbf{B}^{T}\right\| \mathbf{C}^{T}=0
$$

where $\mathbf{E}$ is an unique matrix of $k$ order but $\mathbf{B}$ is an $s \times k$ unknown matrix.

By putting:

$$
\mathbf{C}=\left\|\mathbf{C}_{\sigma} \quad \mathbf{C}_{\boldsymbol{\xi}}\right\|,
$$

where $\mathbf{C}_{\sigma}$ and $\mathbf{C}_{\xi}$ are the $s \times k$ and $s \times s$ matrices respectively, the matrix $\mathbf{B}$ could be calculated from the following equations:

$$
\mathbf{C}_{\xi} \mathbf{B}=-\mathbf{C}_{\sigma}
$$

After determining the matrix $\mathbf{B}$, it is easy to apply the algorithm mentioned above for writing the equations of motion of the system under consideration. By such a way the equations (2.15) can be applied for the Chaplyghin's systems with the constraints of the form either (2.3) or (2.18). 


\section{Examples}

Example 1. Let us write the equations of motion of an uniform sphere which rolls no sliding on a horizontal plane (Fig. 1).

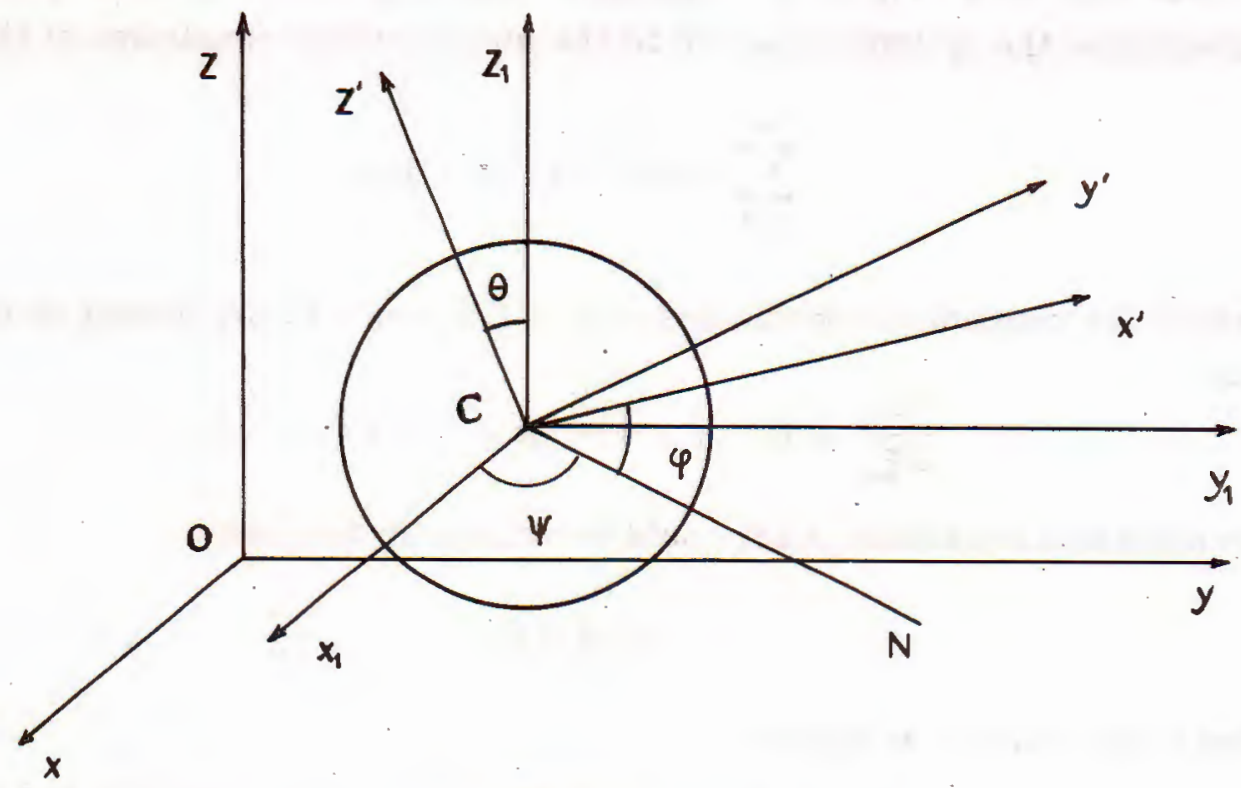

Fig. 1

Let us choose the generalized coordinates by $x, y, \theta, \psi, \varphi$ in which $x, y$ denote the coordinate of the mass center of the sphere but $\theta, \psi, \varphi$ - the Euler angles. The kinetic energy of the sphere is calculated by the formula [6] (see pp. 483-486)

$$
T=\frac{1}{2} J\left(\dot{\theta}^{2}+\dot{\varphi}^{2}+2 \cos \theta \dot{\varphi} \dot{\psi}\right)+\frac{1}{2} m\left(\dot{x}^{2}+\dot{y}^{2}\right) .
$$

Hereafter the following notations are used:

$$
c(\cdot)=\cos (\cdot), \quad s(\cdot)=\sin (\cdot),
$$

$\mathbf{A}(k \times s)$ is the matrix of $k$ rows and $s$ columns i.e. a $k \times s$ matrix.

The condition of the roll without sliding is written as follows:

$$
\begin{aligned}
& \dot{x}=a s \psi \dot{\theta}-a s \theta c \psi \dot{\varphi} \\
& \dot{y}=-(a c \psi \dot{\theta}+a s \theta s \psi \dot{\varphi}) .
\end{aligned}
$$

Since the sphere moves under the action of only the gravitation, the matrix $\mathbf{Q}$ $(5 \times 1)$ is a zero matrix. Evidently, the system under consideration is a Chaplyghin's system of three degrees of freedom with constraints of the form (2.3). 
First, it is to calculate the matrix $\mathbf{G}(5 \times 1)$, which is of the form:

$$
\mathbf{G}^{T}=\|-J s \theta \dot{\varphi} \dot{\psi} \quad J s \theta \dot{\theta} \dot{\psi} \quad J s \theta \dot{\theta} \dot{\varphi} \quad 0 \quad 0\| .
$$

In order to write the equations of motion of the system under considerations, let us apply the equations (2.15).

The matrix of inertia $\mathbf{A}(5 \times 5)$ is of the form:

$$
\mathbf{A}=\left|\begin{array}{ccccc}
J & 0 & 0 & 0 & 0 \\
0 & J & J c \theta & 0 & 0 \\
0 & J c \theta & J & 0 & 0 \\
0 & 0 & 0 & m & 0 \\
0 & 0 & 0 & 0 & m
\end{array}\right|
$$

Let us choose $\theta, \psi, \varphi$ to be the independent coordinates. We have:

$$
\begin{aligned}
& \mathbf{A}_{1}(3 \times 3)=\left\|\begin{array}{ccc}
J & 0 & 0 \\
0 & J & J c \theta \\
0 & J c \theta & J
\end{array}\right\|, \quad \mathbf{A}_{2}(2 \times 2)=\left\|\begin{array}{cc}
m & 0 \\
0 & m
\end{array}\right\|, \\
& \mathbf{A}_{3}(2 \times 3)=\left\|\begin{array}{lll}
0 & 0 & 0 \\
0 & 0 & 0
\end{array}\right\|, \quad \mathbf{A}_{4}(3 \times 2)=\left\|\begin{array}{ll}
0 & 0 \\
0 & 0 \\
0 & 0
\end{array}\right\| .
\end{aligned}
$$

From (3.2) we have:

$$
\begin{aligned}
& \mathbf{B}(2 \times 3)=\left\|\begin{array}{ccc}
a s \psi & -a s \theta c \psi & 0 \\
-a c \psi & -a s \theta s \psi & 0
\end{array}\right\|, \\
& \dot{\mathbf{B}}(2 \times 3)=\left\|\begin{array}{lll}
a c \psi \dot{\psi} & a(s \theta s \dot{\psi} \psi-c \theta s \psi \dot{\theta}) & 0 \\
a s \psi \dot{\psi} & -a(s \theta c \psi \dot{\psi}+c \theta s \psi \dot{\theta}) & 0
\end{array}\right\|,
\end{aligned}
$$

and

$$
\begin{array}{ll}
\mathbf{Q}_{\sigma}(3 \times 1)=\mathbf{O}, & \mathbf{Q}_{\xi}(2 \times 1)=\mathbf{O}, \\
\mathbf{G}_{\sigma}(3 \times 1)=\left\|\begin{array}{c}
-J s \theta \dot{\varphi} \dot{\psi} \\
J s \theta \dot{\psi} \dot{\theta} \\
J s \theta \dot{\theta} \dot{\varphi}
\end{array}\right\|, & \mathbf{G}_{\xi}(2 \times 1)=\mathbf{O} .
\end{array}
$$

Following (2.16), the matrix $\mathbf{A}_{\sigma}(3 \times 3)$ in the equations (2.15) is now:

$$
\begin{aligned}
\mathbf{A}_{\sigma} & =\mathbf{A}_{1}+\mathbf{B}^{T} \mathbf{A}_{2} \mathbf{B}= \\
& =\left\|\begin{array}{ccc}
J & 0 & 0 \\
0 & J & J c \theta \\
0 & J c \theta & J
\end{array}\right\|+\left\|\begin{array}{cc}
a s \psi & -a c \psi \\
-a s \theta c \psi & -a s \theta s \psi \\
0 & 0
\end{array}\right\|\left\|\begin{array}{cc}
m & 0 \\
0 & m
\end{array}\right\|\left\|\begin{array}{ccc}
a s \psi & -a s \theta c \psi & 0 \\
-a c \psi & -a s \theta s \psi & 0
\end{array}\right\| \\
& =\left\|\begin{array}{ccc}
J+m a^{2} & 0 & 0 \\
0 & J+m a^{2} s^{2} \theta & J c \theta \\
0 & J c \theta & J
\end{array}\right\|
\end{aligned}
$$


The matrix $\mathbf{A}^{*}(3 \times 3)$ in the equations (2.15) is calculated by the formula (2.17), that is:

$$
\begin{aligned}
& \mathbf{A}^{*}=\mathbf{B}^{T} \mathbf{A}_{2} \dot{\mathbf{B}}= \\
& =\left\|\begin{array}{cc}
a s \psi & -a c \psi \\
-a s \theta c \psi & -a s \theta s \psi \\
0 & 0
\end{array}\right\|\left\|\begin{array}{cc}
m & 0 \\
0 & m
\end{array}\right\|\left\|\begin{array}{ccc}
a c \psi \dot{\psi} & a(s \theta s \psi \dot{\psi}-c \theta c \psi \dot{\theta}) & 0 \\
a s \psi \dot{\psi} & -a(s \theta c \psi \dot{\psi}+c \theta s \psi \dot{\theta}) & 0
\end{array}\right\| \\
& =\left\|\begin{array}{ccc}
0 & m a^{2} s \theta \dot{\psi} & 0 \\
-m a^{2} s \theta \dot{\psi} & m a^{2} s \theta c \theta \dot{\theta} & 0 \\
0 & 0 & 0
\end{array}\right\|
\end{aligned}
$$

Equations (2.15) are now

$$
\begin{aligned}
& \left\|\begin{array}{ccc}
J+m a^{2} & 0 & 0 \\
0 & J+m a^{2} s^{2} \theta & J c \theta \\
0 & J c \theta & J
\end{array}\right\|\left\|\begin{array}{c}
\ddot{\theta} \\
\ddot{\varphi} \\
\ddot{\psi}
\end{array}\right\|= \\
& =\left\|\begin{array}{ccc}
-J s \theta \dot{\varphi} \dot{\psi} \\
J s \theta \dot{\psi} \dot{\theta} \\
J s \theta \dot{\theta} \dot{\varphi}
\end{array}\right\|-\left\|\begin{array}{ccc}
0 & m a^{2} s \theta \dot{\psi} & 0 \\
-m a^{2} s \theta \dot{\psi} & m a^{2} s \theta c \theta \dot{\theta} & 0 \\
0 & 0 & 0
\end{array}\right\|\left\|\begin{array}{|c}
\dot{\theta} \\
\dot{\varphi} \\
\dot{\psi}
\end{array}\right\|
\end{aligned}
$$

or in the development form:

$$
\begin{aligned}
& \left(J+m a^{2}\right) \ddot{\theta}=J s \theta \dot{\psi} \dot{\varphi}-m a^{2} s \theta \dot{\varphi} \dot{\psi}, \\
& \left(J+m a^{2} s^{2} \theta\right) \ddot{\varphi}+J c \theta \ddot{\psi}=J s \theta \dot{\theta} \dot{\psi}+m a^{2} s \theta \dot{\psi} \dot{\theta}-m a^{2} s \theta c \theta \dot{\theta} \dot{\varphi}, \\
& J c \theta \ddot{\varphi}+J \ddot{\psi}=J s \theta \dot{\theta} \dot{\varphi} .
\end{aligned}
$$

The last equation of (3.12) gives a first integral

$$
c \theta \dot{\varphi}+\dot{\psi}=\text { const. }
$$

The results obtained coincide completely with the ones in [6].

Example 2. Let us consider an automobile moving in a horizontal plane (Fig. 2). The mass, the moment of inertia about the mass centre and the length are $m, J$ and $\ell$ respectively. The force $F$ is acting in the lengthwise axis of the automobile. Let choose $x, y, \varphi$ as the generalized coordinates, i.e. the coordinate of the mass centre and the angle between the lengthwise and $x$ axis. The controlling parameter is $\theta$, i.e. the inclined angle of the front wheel plane with respect to the lengthwise (see [5], pp. 22-31). 


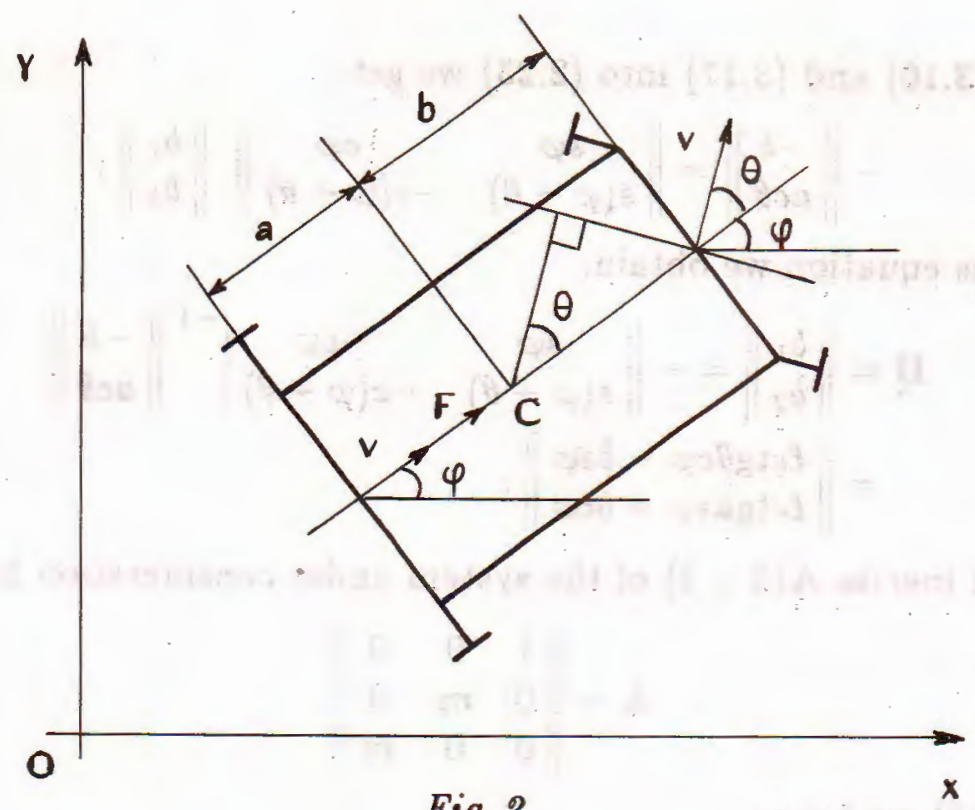

Fig. 2

The kinetic energy of the system could be written as:

$$
T=\frac{1}{2} J \dot{\varphi}^{2}+\frac{1}{2} m\left(\dot{x}^{2}+\dot{y}^{2}\right)
$$

The system under consideration is subjected by two nonholonomic constraints of the form

$$
\begin{aligned}
& \dot{x} s \varphi-\dot{y} c \varphi+b \dot{\varphi}=0, \\
& \dot{x} s(\varphi+\theta)-\dot{y} c(\varphi+\theta)-a c \theta \dot{\varphi}=0 .
\end{aligned}
$$

The generalized forces are now:

$$
Q_{\varphi}=0, \quad Q_{x}=F c \varphi, \quad Q_{y}=F s \varphi
$$

Clearly that the system under consideration is a Chaplyghin's system of one degree of freedom. In connection with this, let us choose $\varphi$ to be independent coordinate. The constraints imposed on the system are of the form (2.16). Thus we concern with the case in which the constraint equations are written in the form unsolved with respect to dependent velocities.

In order to apply the equations (2.15), it is necessary to calculate the matrix $\mathbf{B}$ which is a solution of the equations (2.23). The matrix $\mathbf{B}$ could be written as follows:

$$
\mathbf{B}^{T}=\left\|b_{1} \quad b_{2}\right\|
$$

In accordance with (2.22) we have:

$$
\mathrm{C}_{\xi}(2 \times 2)=\left\|\begin{array}{cc}
s \varphi & -c \varphi \\
s(\varphi+\theta) & -c(\varphi+\theta)
\end{array}\right\|, \quad \mathbf{C}_{\sigma}(2 \times 1)=\left\|\begin{array}{c}
-b \\
a c \theta
\end{array}\right\| .
$$


Substituting (3.16) and (3.17) into (2.23) we get:

$$
-\left\|\begin{array}{c}
-b \\
a c \theta
\end{array}\right\|=\left\|\begin{array}{cc}
s \varphi & -c \varphi \\
s(\varphi+\theta) & -c(\varphi+\theta)
\end{array}\right\|\left\|\begin{array}{l}
b_{1} \\
b_{2}
\end{array}\right\| .
$$

By solving this equation we obtain:

$$
\begin{aligned}
\mathbf{B} & =\left\|\begin{array}{l}
b_{1} \\
b_{2}
\end{array}\right\|=-\left\|\left.\begin{array}{cc}
s \varphi & -c \varphi \\
s(\varphi+\theta) & -c(\varphi+\theta)
\end{array}\right|^{-1}\right\| \begin{array}{c}
-b \\
a c \theta
\end{array} \| \\
& =\left\|\begin{array}{l}
\ell c \operatorname{tg} \theta c \varphi-b s \varphi \\
\ell c \operatorname{tg} \theta s \varphi+b c \varphi
\end{array}\right\| .
\end{aligned}
$$

The matrix of inertia $\mathbf{A}(3 \times 3)$ of the system under consideration has the form:

$$
\mathbf{A}=\left\|\begin{array}{ccc}
1 & 0 & 0 \\
0 & m & 0 \\
0 & 0 & m
\end{array}\right\|
$$

Following (2.13) we have:

$$
\mathbf{A}_{1}(1 \times 1)=\|J\|, \quad \mathbf{A}_{2}(2 \times 2)=\left\|\begin{array}{cc}
m & 0 \\
0 & m
\end{array}\right\|, \quad \mathbf{A}_{3}(2 \times 1)=\mathbf{0}, \quad \mathbf{A}_{4}(1 \times 2)=\mathbf{0} .
$$

Differentiating the matrix $\mathbf{B}$ of (3.19) we obtain:

$$
\dot{\mathbf{B}}^{T}=\left\|-\left(\frac{\ell c \varphi}{s^{2} \theta} \dot{\theta}+\ell \operatorname{ctg} \theta s \varphi \dot{\varphi}+b c \varphi \dot{\varphi}\right) \quad\left(\ell \operatorname{ctg} \theta c \varphi \dot{\varphi}-\frac{\ell}{s^{2} \theta} s \varphi \dot{\theta}-b s \varphi \dot{\varphi}\right)\right\| \text {. }
$$

It is easy to calculate:

$$
\begin{aligned}
& \mathbf{Q}_{\sigma}(1 \times 1)=\mathbf{O}, \quad \mathbf{Q}_{\xi}^{T}(1 \times 2)=\|F c \varphi \quad F s \varphi\| \\
& \mathbf{Q}_{\sigma}(1 \times 1)=\mathbf{O}, \quad \mathbf{G}_{\xi}(1 \times 2)=\mathbf{0} \text {. }
\end{aligned}
$$

The matrix $\mathbf{A}_{\sigma}(1 \times 1)$ and $\mathbf{A}^{*}(1 \times 1)$ in the equations (2.15) are now:

$$
\begin{aligned}
& \mathbf{A}_{\sigma}=\mathbf{A}_{1}+\mathbf{B}^{T} \mathbf{A}_{2} \mathbf{B}= \\
& \|J\|+\|(\ell \operatorname{ctg} \theta c \varphi-b s \varphi)(\ell \operatorname{ctg} \theta s \varphi+b c \varphi)\|\left\|\begin{array}{cc}
m & 0 \\
0 & m
\end{array}\right\| \|\left(\begin{array}{l}
\ell \operatorname{ctg} \theta c \varphi-b s \varphi) \\
(\ell \operatorname{ctg} \theta s \varphi+b c \varphi)
\end{array}\right. \\
& =\left\|J+m\left(\ell^{2} \operatorname{ctg} \theta+b^{2}\right)\right\| \\
& \mathbf{A}^{*}=\mathbf{B}^{T} \mathbf{A}_{2} \dot{\mathbf{B}}= \\
& \|(\ell c \operatorname{tg} \theta c \varphi-b s \varphi)(\ell c \operatorname{tg} \theta s \varphi+b c \varphi)\|\left\|\begin{array}{cc}
m & 0 \\
0 & m
\end{array}\right\| \| \begin{array}{l}
\frac{\ell c \varphi}{s^{2} \theta} \dot{\theta}+\ell c \operatorname{tg} \theta s \varphi \dot{\varphi}+b c \varphi \dot{\varphi} \\
\ell \operatorname{ctg} \theta c \varphi \dot{\varphi}-\frac{\ell}{s^{2} \theta} s \varphi \dot{\theta}-b s \varphi \dot{\varphi}
\end{array} \\
& =\left\|-m \ell^{2} \operatorname{ctg}^{2} \theta \frac{\dot{\theta}}{s^{2} \theta}\right\|, \\
& \mathbf{Q}=0
\end{aligned}
$$




$$
\mathbf{B}^{T} \mathbf{Q}_{\xi}=\|(\ell \operatorname{ctg} \theta c \varphi-b s \varphi) \quad(\ell \operatorname{ctg} \theta s \varphi+b c \varphi)\|\left\|\begin{array}{l}
F c \varphi \\
F s \varphi
\end{array}\right\|=\|F \ell \operatorname{ctg} \theta\|
$$

Equations (2.15) now are written as follows:

$$
\mathbf{A}_{\sigma} \ddot{\boldsymbol{\sigma}}=\mathbf{B}^{T} \mathbf{Q}_{\xi}-\mathbf{B}^{T} \mathbf{A}_{2} \dot{\mathbf{B}} \dot{\boldsymbol{\sigma}}
$$

which takes the form:

$$
\left\|J+m\left(\ell^{2} \operatorname{ctg}^{2} \theta+b^{2}\right)\right\|\|\ddot{\varphi}\|=\|F \ell c \operatorname{tg} \theta\|-\left\|-m \ell^{2} \operatorname{ctg}^{2} \theta \frac{\dot{\theta}}{s^{2} \theta}\right\|\|\dot{\varphi}\|
$$

or in the development form

$$
\left[J+m\left(\ell^{2} \operatorname{ctg}^{2} \theta+b^{2}\right)\right] \ddot{\varphi}=F \ell \operatorname{ctg} \theta+m \ell^{2} \operatorname{ctg}^{2} \theta \frac{\dot{\theta}}{s^{2} \theta} \dot{\varphi} .
$$

By putting

$$
k^{2}=\frac{J+m b^{2}}{m}, \quad f=\frac{F}{m}
$$

and substituting them in (3.29) we have:

$$
\ddot{\varphi}=\frac{\ell \operatorname{ctg} \theta}{k^{2}+\ell^{2} \operatorname{ctg}^{2} \theta}\left(\frac{1}{s^{2} \theta} \dot{\varphi} \dot{\theta}+f\right),
$$

which describes the motion of the system under consideration. The result obtained coincides with the one given in [5].

It is noticed that for obtaining the above result by other methods, for example by the Lagrangian multiplier method or by the Chaplyghin's equations or by the Appell's equations, we have to carry out some complicated calculations $[5,6]$.

\section{Conclusions}

The equations of motion for Chaplyghin's system introduced has two of advantages.

1) The equations are formulated by using only the matrix of inertia in the Lagrangian function which is calculated easily.

2) The algorithm for establishing the mentioned equations is very simple. Especially, the equations obtained are written in the matrix form which is convenient for automatically programming on computers.

This work is completed with financial support of the Council for Natural Science of Vietnam. 


\section{REFERENCES}

1. Bellman P. Introduction to matrix analysis, Mc Graw-Hill Book Company, Inc. New York Toronto London 1960.

2. Do Sanh. The thesis of Doctor of Science. Hanoi University of Technology, Hanoi, 1984 (in Vietnamese).

3. Do Sanh. A form of equation of motion of a mechanical system, Journal of Mechanics, NCNST of Vietnam, No 3, 1995.

4. Do Sanh. A new form of equations of motion of nonholonomic mechanical system. Journal of Mechanics, NCNST of Vietnam, No 3, 1997 (in Vietnamese).

5. Dobronravov V. V. Fundamental of mechanics of nonholonomic systems, "Nauka", Moscow, 1970 (in Russian).

6. Neimark J. I., Fufaev N. A. Dynamics of nonholonomic system, Vyshaia Schkola, Moscow, 1967 (in Russian).

Received July 15, 1999

\section{MỘT DANG PHƯONG TRÌNH CHUYỂN ĐỘNG CƯA CÁC HỆ CHAPLYGHIN}

Hệ Chaplyghin đóng một vai trò quan trọng trong kỹ thuật. Có thể dùng các phương pháp tổng quát trong cơ học giaii tích để thành lập phương trình chuyển động của các hệ này, ví dụ phương trình Lagrange dạng nhân tử, phương trình Appel,... Tuy nhiên việc sử dụng các phương pháp tổng quát như vậy không làm nổi bật được các đặc điểm của các hệ Chaplyghin. Chaplyghin đã đề xuất một dạng phương trình mà để viết nó chì cần dựa vào hàm Lagrange. Các phương trình này được viết đối với biến độc lập (số biến bằng số bậc tự do) và do đó tạo thành hệ phương trình vi phân cấp hai đóng kín đối với các biến này. Tuy nhiên để viết được phương trình Chaplyghin cần phải tính một số chỉ số khá phức tạp. Trong bài báo đã đưa ra một dạng phương trình chị dựa vào hàm Lagrange (chính xác hơn chì dựa vào ma trận quán tính) và có sơ đồ tính toán khá đơn giản, nhờ vào việc ma trận hóa cách viết các phương trình. Dạng phương trình này rất thích hợp cho việc lập trình tự động trên máy tính.

Department of Applied Mechanics

Hanoi University of Technology

1 Dai Co Viet Str., Hanoi, Vietnam

Tel. 84.4.8680469, Fax: 84.4.8692006

Tel. (Home): 8693402 